beauticians, cleaners, a millwright, a waitress and several workers that either decanted or mixed the chemicals.

Discussion $\mathrm{MCI} / \mathrm{MI}$ and $\mathrm{MI}$ are important occupational allergens and should be tested using the correct concentration to avoid misdiagnosing cases. The importance of keeping abreast with what is happening in the field was highlighted by the changes implemented which improved the detection of these allergens.

\section{7b FROM WORKPLACE TO HOME ENVIRONMENT: SPREADING OF MOUSE ALLERGENS BY LABORATORY ANIMAL WORKERS}

H Kube, L Wengenroth*, J Gerlich, G Dietrich-Gümperlein, R Schierl, D Nowak, K Radon. Institute and Outpatient Clinic for Occupational, Social and Environmental Medicine, Munich, Germany

\subsection{6/oemed-2018-ICOHabstracts. 144}

Introduction Laboratory animal workers (LAW) working with mice are exposed to mouse allergens (MA). Exposure to MA can lead to occupational allergies and asthma. If MA are spread to home environments, the longer duration of exposure might increase the risk for allergic symptoms. Little is known about the spreading of MA. This study aimed to assess:

- whether spreading of MA from workplace to home environment takes place,

- which factors increase spreading of MA.

Methods In a cross-sectional study we took dust samples from the homes of 107 LAW and 13 controls. From 90 LAW we took additional dust samples from their working place. Samples were analysed using mus $m 1$ ELISA kits. Through a questionnaire we assessed socio-demographic data, allergies and cleaning habits. In LAW we also assessed types of cages used, work tasks and protective clothing.

Results MA concentration was higher in home environments of LAW (median (ng mus $\mathrm{m} 1$ )=11.3) than in controls (median $=1.1 ; \mathrm{p}=0.016 ;$ Kruskal-Wallis test). The highest workplace MA concentration was found in the scullery (median $=145,0000.0$ ), followed by the changing rooms (median $=10.2)$ and staffrooms (median $=7.5$ ). MA concentration was higher in homes of LAW who fulfilled cleaning tasks (cleaning of cages, floors, etc.) $(p=0.034)$ and who changed their linen at home less than once a month $(p=0.024)$. MA concentration at home was not associated with duration of mouse contact $(\mathrm{p}=0.909)$ and age of sleeping mattress at home $(p=0.649)$.

Discussion Spreading of MA from workplace to home environment takes place. LAWs who fulfilled cleaning tasks were found to have higher MA concentration at home. Special focus should be given to reduce MA concentration during cleaning in laboratory animal facilities.

\section{C INHALED FINE PARTICLES INDUCE ALLERGIC INFLAMMATION IN THE LUNGS}

\footnotetext{
1,2,3 E Kuroda*, 1,2 KJ Ishii. 'Laboratory of Adjuvant Innovation, Centre for Vaccine and Adjuvant Research (CVAR), National Institutes of Biomedical Innovation, Health and Nutrition, Osaka, Japan; ' 2 Laboratory of Vaccine Science, WPI Immunology Frontier Research Centre (IFReC), Osaka University, Osaka, Japan; ${ }^{3}$ Japan Science and Technology Agency (JST), PRESTO, Japan
}

10.1136/oemed-2018-ICOHabstracts. 145
It is known that inhaled fine particles such as particle pollutions are associated with allergic responses. Many reports have shown that some particulates function as adjuvant to enhance immune responses, however its underlying mechanisms are still unclear. Here, we will show how particulates induce immune responses in the lungs.

We used aluminium salt (alum) and crystalline silica as inflammatory particulate. These inflammatory particulates stimulated alveolar macrophage to release interleukin-1 $\alpha$ (IL$1 \alpha$ ) through cell death. We also observed IL- $1 \alpha$ release in the bronchoalveolar lavage fluid (BALF) after intratracheal instillation (i.t.) of inflammatory particulate alum in vivo. Released IL- $1 \alpha$ in the lungs was thought to be a damage-associated molecular pattern (DAMP) and contributed to antigen-specific IgE production in mouse model of particulate-induced allergic inflammation. In addition, we found the lymphoid cluster formations in the lungs after i.t. alum instillation and allergen exposure. This lymphoid cluster was considered to be inducible bronchus-associated lymphoid tissue (iBALT), and we suggest that iBALT formation might be an important role for local IgE responses in the lungs.

These results indicate that inhalation of particulates and allergen exposure induces and exacerbate allergic responses though the unique immune responses in the lungs such as alveolar macrophage death and iBALT formation.

\section{7d BEEKEEPER ALLERGY RISK AND WORK SUITABILITY}

M Di Gioacchino*, M Di Giampaolo, C Petrarca. Unit of Immunotoxicology and Allergy, CSIMET, G. d'Annunzio University, Chieti, Italy

\subsection{6/oemed-2018-ICOHabstracts.146}

Introduction The risk for a beekeeper to become allergic to bee venom is higher than the general population. The possibility that they develop an allergy is greater when rarely bite with $2 / 3$ month interval. On the contrary, when bites are simultaneous or tight over time the probability of allergy decreases. Beekeepers who receive more than 200 stings do not develop venom allergy. In general, subjects developing a reaction limited to the skin have $70 \%$ possibility of a spontaneous recovery. On the contrary, people haveing a systemic reaction tends to show the same or greater reaction when restung.

Discussion Work suitability of bee allergic beekeepers, which had a systemic reaction is a relevant problem for occupational doctors. They should be prescribed appropriate DPI and provided of auto-injectable adrenaline as emergency treatment, sure that they received the necessary training. Furthermore, it is desirable that every allergic subject receives a desensitising treatment, as it is efficacious in $95 \%$ of patients. The treatment will last for 5 years, except for subjects with high basal tryptase who should be treated for a life-time, as the risk of anaphylaxis reappears after the discontinuation of an already effective immunotherapy. The cardiac involvement during anaphylaxis is very frequent with the picture of Kounis Syndrome (KS), characterised by infarction induced by the effects on coronaries of mast cell chemical mediators. In particular, histamine, usually vasodilator agent, can induce vasoconstriction subjects with coronary endothelial lesions. In this situation, the coronary spasm worsens the perfusion of a tachy heart, already with increased blood demand, with a potentially lifethreatening ischemia. Furthermore, in a subject developing a 
KS the administration of adrenaline should be carefully evaluated as adrenaline, having vasoconstriction effects, can worsen the myocardial ischemia.

Conclusions It is our opinion that work suitability cannot be granted to beekeepers who developed KS with.

\section{OCCUPATIONAL CONTACT DERMATITIS}

EA Ozgur*, Y Demıral, AH Cımrın. Dokuz Eylül University Medical School, Occupational Medicine, Izmir, Turkey

\subsection{6/oemed-2018-ICOHabstracts. 147}

Introduction Dermatoses are a group of diseases that are common among occupational diseases. Occurrence is seen between $10 \%-40 \%$ and is the second most common occupational disease after muscular and skeletal system diseases. In this study, we aimed to evaluate the general characteristics of the occupational diseases evaluated by occupational dermatosis in our clinic about 3 years.

Methods A total of 23 patients who applied between 2014-2017 were evaluated retrospectively. A detailed business story was taken from the cases and clinical evaluation was done. Materials MSDS information was requested from the workplaces. Patch test was applied to the cases where contact dermatitis was suspected. Other dermatological diseases were excluded and evaluated as allergic and irritant contact dermatitis according to the patch test results. Statistical evaluations were performed using the PASW Statistics for Windows (SPSS Inc. Version 18.0, Released 2009, Chicago, USA) statistical package program.

Results When the demographic characteristics were examined, $21.74 \%$ of the cases were female and $78.26 \%$ were male. The initial area of the lesions was 52\%. Six of the cases were diagnosed with other dermatological diseases, 12 with allergic contact dermatitis and 4 with irritant contact The most common allergens were nickel sulphate $(73.3 \%)$, potassium dichromate (40\%) and tiuram (20\%). Time interval between onset of lesions and the date they first appeared (latent periods) according to the sectors is given in the tables.

Conclusion Occupational dermatoses refer to our clinic, constitute $3.7 \%$ of occupational disease-related cases and 5 th place among other occupational diseases. It was observed that the cases came from different sectors, mostly male and between 30-40 years old. Occupational history and patch test results are important for diagnosis. Since the latent period is variable, it is not very meaningful in predicting the occurrence of the disease when examined in terms of substance and sectoral distribution.

\section{AIRBORNE POLLEN SAMPLING AT TWO DIFFERENT HEIGHTS: VARIATION OF CONCENTRATIONS IN INDOOR AND OUTDOOR ENVIRONMENTS AND IMPLICATION FOR OCCUPATIONAL HEALTH}

${ }^{1} \mathrm{P}$ Capone, ${ }^{1} \mathrm{~L}$ Boccacci, ${ }^{1} \mathrm{~S}$ Di Renzi, ${ }^{1} \mathrm{R}$ Ferrante, ${ }^{2} \mathrm{MA}$ Brighetti, ${ }^{3} \mathrm{MC}$ Serra, ${ }^{1} \mathrm{R}$ Sisto, ${ }^{1}$ A Pelliccioni, ${ }^{2}$ A Travaglini, ${ }^{1} M C$ D'Ovidio*. 'National Workers' Compensation Authority (INAIL), Department of Occupational and Environmental Medicine, Epidemiology and Hygiene, Monte Porzio Catone (Rome), Italy; ${ }^{2}$ University of Rome 'Tor Vergata', Department of Biology, Rome, Italy; ${ }^{3}$ Research Unit for Oenology in Central Italy (CREA ENC), Velletri (Rome), Italy

10.1136/oemed-2018-ICOHabstracts. 148
Introduction Allergic respiratory diseases, especially rhinitis and asthma, is of relevant importance within emerging occupational diseases in the last decades. It is therefore fundamental monitoring airborne pollen concentrations for allergic disease prevention. Many factors are able to influence airborne pollen concentrations: meteorological parameters (air temperature, relative humidity, wind speed), seasonal variations, geographical location, human habits and activity. In this context, for research purposes, we have carried out samplings at two different heights to investigate variations of concentrations of several types of airborne pollen in indoor and outdoor environments according to standard methods of the UNI 11108/ 2004 and following.

Methods Airborne pollen grains have been collected using a volumetric sampler, Lanzoni VPPS 2000 type Hirst based on impact collection of atmospheric biological particles. Sampling have been performed in one room office using two samplers located to different heights of the suction nozzle (at $1,10 \mathrm{~m}$ and 0,6 m) during 2016-2017 and in outdoor environment (urban area) using two same samplers situated to two different level (at ground level in Trajan Forum and at $45 \mathrm{~m}$ on ground level on Calandrelli Tower) during 2002-2003. Monitoring stripes were cut and stained with glycerine jelly with fuchsine and then observed with optical microscope to $40 \mathrm{X}$.

Results We observed that pollen concentration are higher at low altitude in both indoor and outdoor samplers. Normally, the outdoor pollen concentration at low level is six or seven times higher than at high altitude, while the indoor pollen is about two time higher.

Conclusion The versatility of sampling at different heights is a very valuable tool to explain how many factors both environmental and personal influence space-time distribution and turbulence related to aerodynamics of these biological particles. The results suggest that pollen gradients at lower level is about five time greater than in indoor respect to outdoor sites.

\section{OCCUPATIONAL IMMEDIATE ALLERGIES TO CHEMICALS}

Eva Helaskoski*, Hille Suojalehto, Kristiina Aalto-Korte. Finnish Institute of Occupational Health, Helsinki, Finland

\subsection{6/oemed-2018-ICOHabstracts. 149}

Introduction Exposure to chemicals occurs in a wide range of professional fields. Chemicals typically induce delayed, cell-mediated sensitisation, but some chemical agents are able to induce immediate-type allergy. The mechanisms of immediate sensitisation to chemicals are still mainly unknown, but specific IgE seems to play a role, at least for some chemical groups. The aim of our study was to evaluate the diagnostic procedures of occupational immediate allergic diseases caused by chemicals and assess the feasibility of skin testing in the diagnostics.

Methods We retrospectively reviewed the patient files of the Finnish Institute of Occupational Health for the period of 1991-2011 to find patients diagnosed with immediate sensitisation to chemicals. We collected data on occupation, exposure, work-related symptoms, clinical and immunological test results, and occupational allergic diseases.

Results Positive skin prick tests (SPT) were noted for organic acid anhydrides, diisocyanates, persulfates, epoxy resin, chlorhexidine and aziridine. Amine hardeners and formaldehyde induced multiple small indifferent reactions. For several other 